\title{
Crowdfunding Astronomy Research With Google Sky
}

\author{
Travis S. Metcalfe, White Dwarf Research Corporation, USA
}

\begin{abstract}
For nearly four years, NASA's Kepler space telescope searched for planets like Earth around more than 150,000 stars similar to the Sun. In 2008 with in-kind support from several technology companies, our non-profit organization established the Pale Blue Dot Project, an adopt-a-star program that supports scientific research on the stars observed by the Kepler mission. To help other astronomy educators conduct successful fundraising efforts, I describe how this innovative crowdfunding program successfully engaged the public over the past seven years to help support an international team in an era of economic austerity.
\end{abstract}

Keywords: Astronomy Education; Kepler; "Pale Blue Dot Project”; “Adopt A Star”; Crowdfunding

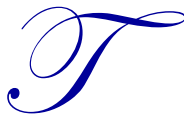

he Kepler space telescope was launched in 2009 and spent the next four years monitoring the brightness of more than 150,000 stars in the constellations Cygnus and Lyra. The mission was designed not only to discover planets around other stars by searching for periodic dips in brightness caused by fortuitously aligned orbits, but also to characterize the parent stars of the newly discovered planetary systems using stellar seismology. As a cost-saving measure prior to launch, in 2004 NASA dropped all financial support for stellar seismology and forged a partnership with a large collaboration led by scientists in Denmark (Gilliland, 2015). In exchange for influence on the selection of targets and early access to proprietary observations, the team provided data analysis services at no cost to NASA. The high-profile agreement gave many European scientists an advantage to secure funding from their home countries, but left US participants largely without support.

As an early-career researcher in 2007, I submitted a proposal to NASA's Kepler Participating Scientist program to help fund my involvement in the Danish-led collaboration. The idea behind the program was to augment the Kepler science team with new expertise that could significantly enhance the scientific return of the mission. Although a panel of independent reviewers recommended my proposal for funding, the Kepler science team decided not to support it. Seeking an alternative source of support for our work, in January 2008 we started the Pale Blue Dot Project (http://adoptastar.whitedwarf.org/) (Metcalfe, 2009).

\section{GUIDING PRINCIPLES \& IMPLEMENTATION}

For-profit companies have been offering informal "name a star" services for decades, the most notable being the International Star Registry. In stark contrast, the Pale Blue Dot Project was designed to compete against these companies while adhering to several guiding principles more consistent with tacit perspectives of the broader scientific community. First, we wanted to be intellectually honest about what we were providing, so we branded our program an "adopt a star" service to dissuade the notion that we were somehow selling stars. This approach is analogous to an "adopt a highway" program that seeks local sponsors to maintain clean roads, where it is well understood that the sponsor does not actually own the road or get to rename it. Instead, a sign along the side of the road acknowledges their support. Second, our program is run by a non-profit organization with $100 \%$ of the proceeds supporting astronomy research. This was only possible with in-kind support from several technology companies and thousands of volunteer hours over the past seven years. Finally, the program sought to establish a direct link between the fundraising mechanism and the science that it supports. We only made the Kepler target stars available for adoption, and the funds generated by the program support research on that same set of stars. 
Now in its eighth year of operations, the Pale Blue Dot Project allows anyone to adopt a Kepler target star for a $\$ 10$ USD donation to support scientific research. Each donor receives a personalized "Certificate of Adoption" by email, with a name or dedication of their choice and a unique star number from the Kepler Input Catalog (Brown, et al., 2011). We update the target on our website with the name of the donor, ensuring that each star can only be adopted once. The Kepler target list was not publicly available when we started the program, so we encouraged "early adopters" to donate without selecting a specific star and gave them the first choice of targets as soon as the catalog was released.

The centerpiece of our outreach and crowdfunding effort was a representation of the Kepler target list in Google Sky, allowing potential donors to browse the locations and basic properties of the stars that NASA was searching for planets. As shown in Figure 1, Google Sky uses a markup language to project icons and other information on top of high-resolution images derived mostly from digitized Palomar Sky Survey photographic plates (Connolly et al., 2008). Any attempt to display all 150,000 targets simultaneously would slow down the computer and ultimately crash the browser. What we needed was a recipe for initially marking the brightest stars, and then gradually highlighting the fainter stars as the user zooms into the field. The open-source regionator software (https://code.google.com/p/regionator/) developed at Google provided exactly the functionality that we needed. It was even distributed with a sample application to display the Swiss national rail network in Google Earth, starting with connections between the busiest train stations but eventually showing all of the connections as the user zooms in. We simply replaced train stations with stars, and used brightness to prioritize the targets.

Figure 1. A representation of the Kepler target list in Google Sky.

Fainter targets are marked as the user zooms in to individual regions of the telescope field of view (blue boxes). Adopted stars are marked with red icons, while available targets are marked with green icons.

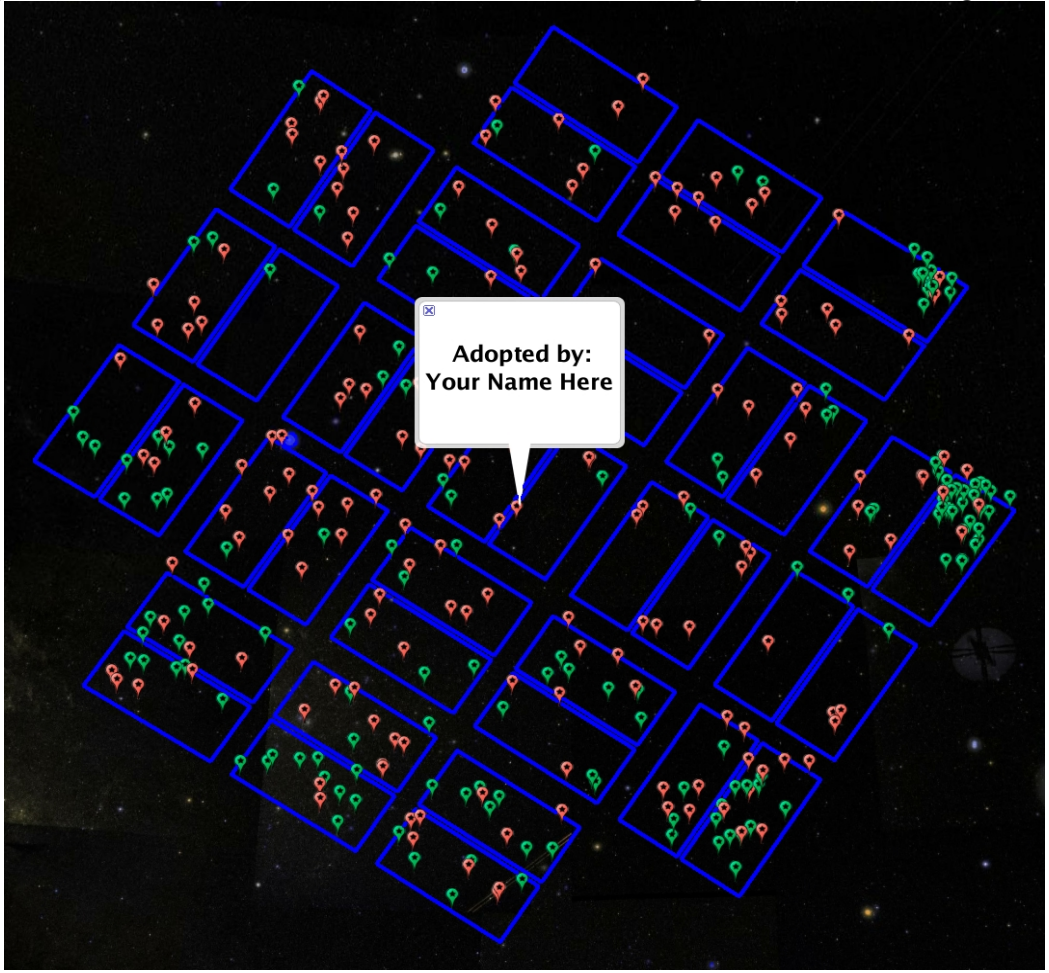

\section{SUCCESSES \& CHALLENGES}

Over the past seven years the Pale Blue Dot Project has gradually grown and is attracting hundreds of donations each month. The history of weekly website visits and star adoptions is shown in Figure 2. For the purposes of the figure, a visit is defined as a session with a duration of up to 30 minutes and at least one interaction (e.g. clicking a link). Sessions that access a single page without interaction typically outnumber these visits by a 
factor of four. Star adoptions account for the total number of successful donations without regard to their value. In 2012 we began offering star adoptions with different donation amounts for value-added targets: $\$ 15$ for double stars from the Kepler eclipsing binary catalog (Prsa, et al. 2011), \$25 for stars with suspected planets from the NASA exoplanet archive (http://exoplanetarchive.ipac.caltech.edu/) and $\$ 100$ for stars with confirmed planets and an official Kepler number. Prior to 2012, our strategy involved driving more traffic to the website to attract more donations. Since then our focus has shifted to converting a higher fraction of our visitors into donors.

Figure 2. Weekly visits and star adoptions during the first seven years of our crowdfunding campaign.

Icons mark significant peaks in web traffic from traditional media coverage $(\equiv)$, social media exposure (@), and annually during Christmas $(\Delta)$ and Valentine's Day $(\boldsymbol{\vee})$.

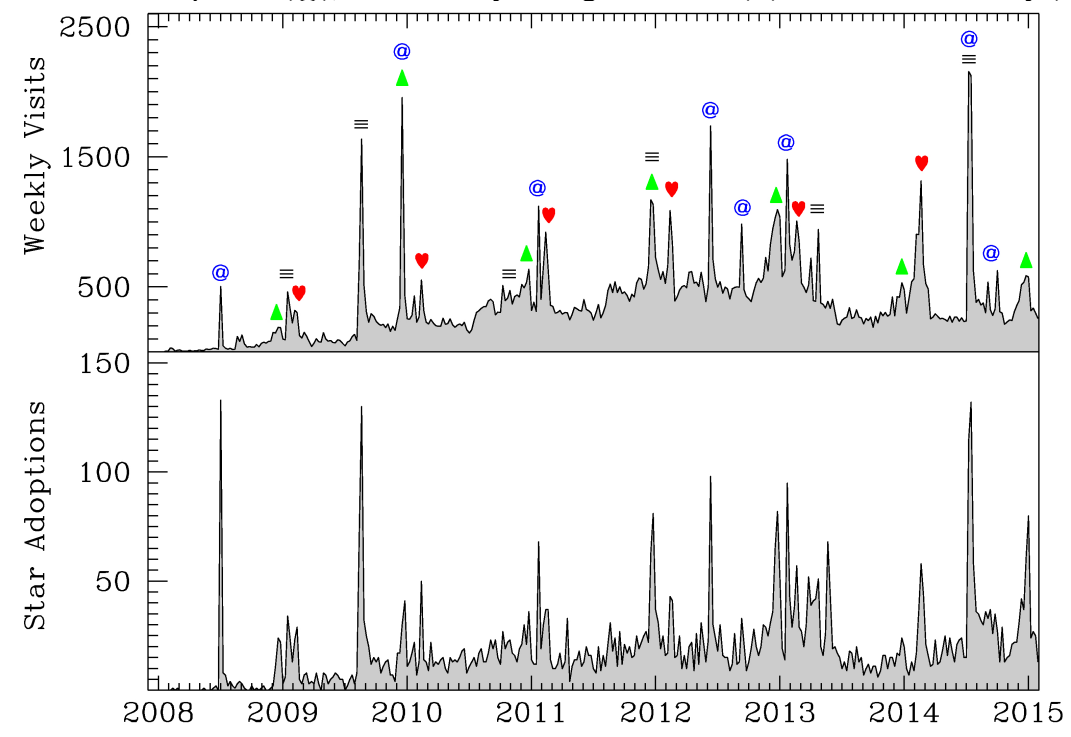

The history of our crowdfunding program is punctuated by recurring features and episodic spikes in both visits and star adoptions. The recurring features correspond to popular gift-giving holidays (Christmas and Valentine's Day). The episodic spikes arise from two sources of publicity: (1) traditional media coverage of our program, generally associated with published scientific results that we supported, and (2) social media exposure, often initiated by enthusiastic donors. Although there is a strong correlation between visits and donations, the dependence is clearly non-linear. Strategically timed publicity can raise awareness of the program during the already-popular seasons. Sometimes this leads to an increase in visits without a comparable boost in donations (as with the social media exposure in late 2009), while other times it has a disproportionate impact on donations (as with the traditional media coverage of Fellet, 2011).

There is an interesting story behind almost every spike in star adoptions, and it is worth elaborating on the largest few because they were associated with some of the greatest challenges to our program. The first major spike in mid-2008 was the result of a short post on the tech news website Slashdot (http://science.slashdot.org/story/08/07/01/1724256/adopt-a-star-to-fund-research). In 2008 the concept of crowdfunding was still in its infancy, and popular platforms like Kickstarter didn't yet exist. This novelty and the strong appeal to the interests of Slashdot readers led to the highest rate of donations in the history of the project, with roughly one in three visitors proceeding to adopt a star. We had certainly found our constituency, but we also attracted the attention of the Kepler science team.

Somewhat unexpectedly, a few days after the Slashdot post appeared, we received an email from NASA's Principle Investigator for the Kepler mission, William Borucki. He expressed some concerns about whether we planned to allow star adoptions only for the Kepler targets, and questioned our intentions for how the funding would be used. He requested that we include a disclaimer on the website to inform visitors that our program was "not authorized or endorsed by NASA". We added a disclaimer to the project website, but the issue prompted one 
member of our board of directors to resign, and we ultimately reconstituted our guiding board with younger colleagues who were more comfortable with the concept of crowdfunding.

The second large spike in star adoptions came from traditional media coverage of a press release about our program in August 2009. An initial article was published two days after the press release by Space.com (Moskowitz 2009), with an additional article by New Scientist (Grossman, 2009) a few days later. Both articles included links to our website, making it easy for readers to support the program. The impact of this media coverage exceeded the Slashdot post because the response was sustained over a longer period of time. Shortly after the New Scientist article appeared, we received an email from a representative of the Carl Sagan Foundation. The article had included the following sentence: "The programme ... is called 'Pale Blue Dot' to echo Carl Sagan's description of Earth as seen from space." The representative insisted that the name of our project constituted an unauthorized use of copyrighted material, since 'Pale Blue Dot' was the title of a book written by Carl Sagan in 1994. Six weeks later, we received a "cease and desist" letter from an attorney representing the Sagan estate. We spent a month working with a pro bono lawyer to refute their illegitimate claim to the phrase (book titles are not protected by copyright law).

The largest surge in star adoptions during the history of our project came in the middle of 2014, when Ukrainian astronomers dedicated a star to an unflattering nickname for Russian president Vladimir Putin. As intended, our \$10 USD star adoptions were undercutting the prices of for-profit competitors. This motivated independent operators in several countries to market Kepler star adoptions in their native language, adopting stars through our website and then reselling them to their customers with additional services. A political group in Ukraine had adopted a star under the controversial name through one of these operators, and then began publicizing it through social media in late June 2014. By early July the insult had gone viral, with an image of the Ukrainian "Certificate of Adoption" guiding supporters to the Pale Blue Dot Project. On the morning of July 4 that year, I received a telephone call from a reporter at the Moscow Times asking for comment (http://www.themoscowtimes.com/news/article/no-plans-to-rename-star-called-putin-is-a-dckhead/503017.html). I told him "Free speech is now written in the stars. We have no plans to censor any of these star adoptions. We appreciate the support for science." Several patriotic Russians were disappointed with our decision.

\section{LESSONS LEARNED}

Although the Google Sky interface to the Kepler target list serves as a highly useful public outreach tool, the interface sometimes proved to be a frustrating technical obstacle for many visitors. To view it through our website, users were required to install the Google Earth plugin on their Internet browser. The plugin was available for the most common desktop operating systems, but there was no support under Linux or for mobile devices. Looking for an easier way to select a star, Dutch software developer Sjors Provoost imported the Kepler target list into a database and created a simple interface to search it and to sort the results. One consequence of his approach was that each star in the database had its own webpage, where he included an image of the star using Google Maps along with coordinates and other basic properties from the Kepler Input Catalog. When he finished, he uploaded the software to an open-source repository and helped us reconfigure it for our program (https://github.com/Sjors/paleblue-dot/tree/master). We added social media share buttons, a link to the "Certificate of Adoption", the option to switch between Google Maps and Sky, and a list of nearby Kepler targets. An example is shown in Figure 3, the most-shared star in our database, which was adopted for a member of the Korean pop band EXO.

The impact of offering an easier method of selecting a star through the database reinforced the most important lesson we have learned from our crowdfunding program: keep it simple. Most of our visitors seem to fall into one of two main categories: (1) "enthusiasts" who are excited about the science we do and are happy to support us, and (2) "customers" who are looking for a unique gift delivered immediately at a low price. The first group is interested enough to read the text on our website, browse the Kepler stars in Google Sky, and support our science with larger donations for the value-added targets. The second group wants the process to be intuitive enough to complete by following visual cues, they are not so interested in the specific star they adopt, and they are less likely to donate more than the minimum. Serving the needs of both groups has made our program more appealing to all visitors. We minimize text up front, but we include links to more information for those who want additional details. Access to the database makes it easy to select a star with specific characteristics, but we assign the brightest star 
available by default so no selection is required. Value-added targets create an incentive for generosity, but large numbers of small donations still help.

Figure 3. An example star page from the Pale Blue Dot Project database, where users can share the star adoption on social media, download their "Certificate of Adoption", view an image of the star in Google Maps or Sky, and see a list of nearby stars to adopt for friends.

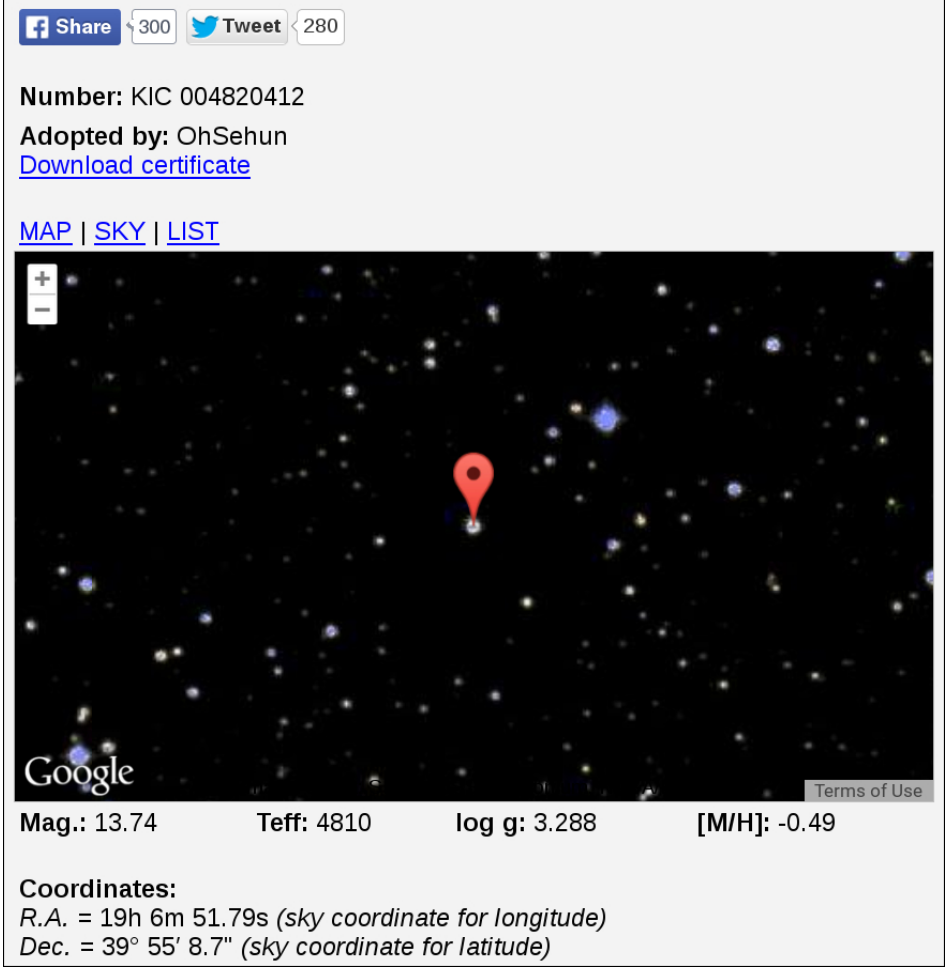

A generation ago, it was not uncommon for scientists to rely on other professionals for some of the things that we now do for ourselves. For example, in the past, there were software engineers to write computer code, drafters to create camera-ready figures, secretaries to edit and publish manuscripts. Today, desktop computers make it easier for scientists to be more self-reliant, but the fact that we can learn how to do web development, database management, and online marketing doesn't make us the most qualified for the job. The final lesson we learned from the Pale Blue Dot Project is to hire consultants for the most important work. We brought in an experienced web developer to redesign the website in 2014 , and annual donations nearly doubled. We are now working with a professional marketing firm to overhaul our online advertising strategy. In the first seven years of the program we attracted about $\$ 100,000$ in donations, but we brought in half of this total in just the past two years. Relatively small investments in people who do not think like scientists can reap benefits that quickly exceed their cost, giving us more time and funding for our research.

\section{FUTURE OUTLOOK}

When we started the Pale Blue Dot Project seven years ago, we envisioned quickly raising $\$ 1.5$ million USD and establishing an endowment for our research over the lifetime of the Kepler mission. In a different universe, it might have played out that way. One of our "early adopters" in 2008 knew a writer for the television show The Colbert Report, and pitched an idea for a segment about our project. In 2010 we were contacted by a marketing firm looking to celebrate the $25^{\text {th }}$ anniversary of the Super Mario Brothers video game by adopting a constellation of Kepler stars in the shape of Mario's face. Neither of these opportunities came to fruition, but we still raised enough funding over the years to help students and early-career scientists present their research at annual workshops, and to help some of our colleagues in developing countries pay the publication charges for their research papers. 
With all of the infrastructure in place and a well-established brand, there is enormous potential for future missions to benefit from our crowdfunding program. The Transiting Exoplanet Survey Satellite (TESS) will launch in 2017, and it will do for the brightest stars in the sky what Kepler did for one small patch of the summer Milky Way. The European PLATO mission will do even more for stellar seismology starting in 2024, with extended observations all around the Galactic plane. Both missions will rely on international teams to help analyze the observations. With foresight and cooperation from these missions, citizens around the globe are able to support this exciting work through the Pale Blue Dot Project. In the end, we judge this strategy to hold substantial promise for similar, future efforts bringing scientists and citizens together to support valuable scientific work.

\section{ACKNOWLEDGMENTS}

The Pale Blue Dot Project has received in-kind support from Google, PayPal, Dreamhost, and SmartClick Adworks. We would like to thank Sjors Provoost, Ian Shorrock, and Robert Piller for contributions that substantially improved our crowdfunding program. This work would not have been possible without support from the Stellar Astrophysics Centre at Aarhus University in Denmark. Earlier versions of this manuscript have been posted online at arXiv.org and ResearchGate.net

\section{AUTHOR INFORMATION}

Travis S. Metcalfe is the director of White Dwarf Research Corporation, and a research scientist based in Boulder, Colorado. He started the Pale Blue Dot Project in 2008, and he has volunteered thousands of hours for daily operations over the past seven years. He hopes to see crowdfunding support for more astronomy projects in the future, and he is open to collaboration with like-minded individuals.

\section{REFERENCES}

Brown, T.M., Latham, D.W., Everett, M.E., \& Esquerdo, G.A. (2011). Kepler Input Catalog: Photometric Calibration and Stellar Classification. The Astronomical Journal, 142, 112.

Connolly, A., Scranton, R., \& Ornduff, T. (2008). Google Sky: A Digital View of the Night Sky. Astronomical Society of the Pacific Conference Series, 400, 96.

Fellet, M. (2011). Crowdfunding science: Give a gift to research. New Scientist. (http://www.newscientist.com/blogs/culturelab/2011/12/give-a-gift-to-research.html).

Gilliland, R.L. (2015). Prelude to, and Nature of the Space Photometry Revolution. European Physical Journal Conference Series, in press (http://arXiv.org/abs/1411.2531).

Grossman, L. (2009). Stars put up for adoption to fund exoplanet research. New Scientist. (http://www.newscientist.com/article/dn17622-stars-put-up-for-adoption-to-fund-exoplanet-research.html).

Metcalfe, T.S. (2009). The Pale Blue Dot Project: an Adopt-a-star Program to Fund Research. Bulletin of the American Astronomical Society, 41, 410.

Moskowitz, C. (2009). Adopt a Star, Help Fund Science. Space.com (http://www.space.com/7135-adopt-star-fundscience.html).

Prsa, A. et al. (2011). Kepler Eclipsing Binary Stars. I. Catalog and Principal Characterization of 1879 Eclipsing Binaries in the First Data Release. The Astronomical Journal, 141, 83. 